\title{
Testosterone, dihydrotestosterone, and spatial task performances of males
}

\author{
WALTER F. MCKEEVER \\ Northern Arizona University, Flagstaff, Arizona \\ and \\ RICHARD A. DEYO \\ Northwestern University, Evanston, Illinois
}

\begin{abstract}
Fifty-eight male college students were administered two spatial tests-the Minnesota Paper Form Board (MPFB) and the Stafford Identical Blocks Test (SIBT). Testosterone (T) and dihydrotestosterone (DHT) levels extant during testing were determined from four blood samples collected over the course of testing. Neither T nor DHT showed significant relationships to spatial ability, but the ratio DHT/T was significantly correlated with the MPFB scores. Subjects whose $\mathrm{T}$ and DHT values deviated most from the group means had significantly higher mean spatial test scores. The DHT/T deviation scores and T deviation scores were uncorrelated and together accounted for about $15 \%$ of the variance in the composite spatial ability scores. Given the inconsistency of previous findings regarding a relationship of $T$ to spatial ability, the present results suggest that future studies should assess DHT as well as $T$, and that data should be carefully examined for possible curvilinear relationships as well.
\end{abstract}

A sex difference in spatial ability, favoring males, is apparent after puberty (Maccoby \& Jacklin, 1974; McGee, 1979). It appears that this sex difference cannot be wholly accounted for by socialization factors (Harris, 1981). Thus, a biological basis has been sought, and the possibility that the explanation may be found in gonadal hormone differences has received attention. The data, however, are disconcertingly contradictory.

Most data favoring a relationship of gonadal hormones to spatial ability is derived from studies in which hormone levels were inferred rather than measured. These include studies of women at different phases of the menstrual cycle (Broverman, Vogel, Klaiber, Majcher, \& Paul, 1981; Hampson \& Kimura, 1988) and investigations in which inferences regarding the level of "male" hormones or "androgens" were made from secondary sex characteristics (Broverman, Broverman, Kobayashi, \& Palmer, 1964; Petersen, 1976).

Studies assessing menstrual-cycle phase suggest that spatial ability is higher in females tested at a point thought to correspond to low levels of gonadal steroids (during or immediately after menses) than at a point thought to correspond to high levels of gonadal steroids (near ovulation). Gordon and Lee (1986), however, found no consistent relationship between assayed levels of estrogen, progesterone, follicle stimulating hormone (FSH), or leutinizing hormone (LH) and spatial ability in women.

\footnotetext{
Supported in part by Grant NS10214-17 from the NINCDS. Correspondence may be addressed to Walter F. McKeever, Department of Psychology, Northern Arizona University, Flagstaff, AZ 86011-5106.
}

Studies of the relationship of secondary sex characteristics, thought to reflect the action of male hormones or androgens, suggest that highly androgenized males perform significantly less well on spatial tests than do less androgenized males (Broverman et al., 1964; Petersen, 1976). Petersen (1976) also studied females, and found that the more androgenized females, defined in terms of secondary sex characteristics, were superior in spatial ability. According to this data, androgenization is favorable for spatial ability of females, but is unfavorable for males. Berenbaum and Resnick (1982), however, in a study generally similar to that of Petersen, found little support for such relationships.

Shute, Pellegrino, Hubert, and Reynolds (1983) attempted to relate levels of circulating testosterone (T) to performances on a number of spatial tests. Their subjects were college students. The antibody employed, however, was not very specific for $T$, since the obtained values were much too high to represent T. For this reason, Shute et al. referred to their independent variable as "androgen" rather than $T$. This measure showed a significant negative relationship to spatial ability in males, and a nonsignificant trend for a positive relationship in females. In a second experiment, Shute et al. contrasted androgen levels of the six highest and six lowest spatial ability subjects selected from a new sample of 33 subjects. A significant interaction of sex and androgen level was found for the Minnesota Paper Form Board (MPFB; Likert \& Quasha, 1941). Females who were high and males who were low on the androgen measure were superior in spatial ability. A second ANOVA was applied to the mean scores of two spatial tests that required mental rotation. These were from 
the Primary Mental Abilities Test (Thurstone, 1965) and the Comprehensive Ability Battery (Hakstian \& Cattell, 1976). The results were in the same direction as for the MPFB, but were not significant. Consistent with the view of Petersen (1976), the authors concluded that spatial ability was negatively related to androgens in males and positively related to androgens in females, with the effect being clearer in males.

Other studies in which $\mathrm{T}$ has been specifically assayed have not supported this relationship, however. Gordon and Lee (1986) examined the relationship of T (and FSH and $\mathrm{LH}$ as well) to performances on a battery of brief spatial tasks. Their subjects were 32 young men who took the same tests 1 week apart. In the first test session, the average $T$ level during the session was found to be positively related to a spatial test requiring mental rotation $(r=+.54)$, with a nonsignificant positive correlation of $T$ with the composite $z$ score of the four spatial tasks administered. In the second session, however, no significant correlation between spatial task performances and $\mathrm{T}$ was seen. Only FSH showed a relationship to the composite spatial ability score in both test sessions, the correlations being -.52 and -.53 , respectively. McKeever, Rich, Deyo, and Conner (1987) correlated scores from the MPFB, the Stafford Identical Blocks Test (SIBT; Stafford, 1961), the Shipley Hartford Vocabulary Test (Shipley, 1940), and a brief test of word fluency with $T$ levels. No relationship of $T$ to performance on any of the individual tests or combinations of the tests was found.

Christiansen and Knussman (1988) reported a study challenging the thrust of the findings from Broverman's group and the other studies that had suggested that androgens are negatively related to spatial ability in males. They examined the relationships between spatial ability scores and five hormone measures [plasma $\mathrm{T}$, dihydrotestosterone (DHT), salivary $\mathrm{T}, \mathrm{DHT} / \mathrm{T}$, and salivary $\mathrm{T} /$ plasma $\mathrm{T}$ ]. A number of significant, though small (all in the range from .19 to .26), correlations were found. These were always positive correlations for plasma $\mathrm{T}$, DHT, and salivary $\mathrm{T}$, and negative correlations for the DHT/T and salivary T/plasma $\mathrm{T}$ ratios; that is, for the latter ratio measures, higher levels of plasma $T$ to DHT and to salivary T, respectively, were favorable for spatial ability. Christiansen and Knussman argued that Broverman et al. (1964) and Petersen (1976) employed faulty assumptions about the relationship of $T$ to secondary sex characteristics. Christiansen and Knussman saw their results as bringing the global relevant data into congruence-that is, explaining the sex difference in spatial ability, the usually found impairment in spatial ability among hypogonadal males, and the within-sex differences in spatial ability of normal males and females. In short, the more androgens, the higher the spatial ability. It must be noted, however, that neither Gordon and Lee (1986) nor McKeever et al. (1987) found dependable relationships of $\mathrm{T}$ to spatial abilities.
The present paper presents new data on the relationship of T and DHT to spatial ability.

\section{METHOD}

\section{Subjects and Procedure}

The subjects were 58 undergraduate males at Northern Arizona University who received both introductory psychology course-requirement credit and $\$ 10$ for their participation. The subjects were given a brief medical screening questionnaire to rule out neurological diseases or traumatic brain injury, and then the SIBT and MPFB were administered, in that order. The SIBT is a 30-item test in which the subject must decide which of five choice "blocks," represented as three-dimensional, is the same shape as a test block, despite the rotation of the choice block to a different orientation. The MPFB is a 64 -item test requiring the subject to decide which of five configurations could be constructed from components shown in a test item. The MPFB is more a "spatial relations" task than the SIBT and requires little mental rotation. The two tests show only moderate correlation $(r=.40, p<.002$ in the present sample). Time limits of 15 and $20 \mathrm{~min}$, respectively, were imposed for the SIBT and MPFB. Subjects were scheduled for sessions that began at either 3:00 p.m. or 4:00 p.m. Thus, 29 subjects were tested in the 3:00 p.m. session and 29 in the 4:00 p.m. session. Analysis of T, DHT, and the spatial tests for possible effects of time of testing showed no significant effects.

Four blood samples, from finger-tip punctures, were collected in heparinized capillary tubes. The taking of multiple samples across the test session is desirable given the known pulsatile release of $T$. The first sample was taken after the screening questionnaire and some general instruction regarding the necessity of the blood samples (the subjects had been told of this prior to the experimental session as well). Subsequent samples were taken at approximate 20 -min intervals. Samples were spun at 2,500 rpm for $7 \mathrm{~min}$. Nine of the 232 samples were lost due to breakage of capillary tubes during centrifuging. All four samples were available for 49 of the 58 subjects; each of the remaining 9 subjects had three samples intact. Plasma samples were frozen at $-23^{\circ} \mathrm{C}$ until assayed.

\section{T/DHT Assay}

Concentrations of $\mathrm{T}$ and DHT were determined using a commercially available kit (Amersham Corp.). Standards (12.5, 25, 50, 100, and $200 \mathrm{pg} \mathrm{T} ; 25,50,100,200$, and $400 \mathrm{pg}$ DHT), samples, antiserum, and $\mathrm{DHT}^{[\mathrm{H} 3]}$ were brought to a total volume of $1.0 \mathrm{ml}$ using distilled water in $12 \times 150 \mathrm{~mm}$ clean glass test tubes. The samples were extracted by adding $3 \mathrm{ml}$ diethyl ether and vortexing. The ether extracts were combined and evaporated to dryness under a gentle stream of air. The residue was dissolved by adding $2.5 \mathrm{ml}$ of assay buffer and vortexed for $10 \mathrm{sec}$. The sample was then divided into 2 samples $(0.4$ and $2.10 \mathrm{ml}$, respectively). The larger sample was oxidated to remove $\mathrm{T}$ prior to the determination of DHT.

Separate standard curves were calculated for the total steroid content ( $\mathrm{T}+\mathrm{DHT}$ ) using the unoxidated samples, and for the DHT concentration (using oxidated samples). All samples and standards were run in duplicate. Two hundred microliters of each standard or sample was added, followed by $200 \mu$ of tritiated DHT to each tube, followed by the addition of antiserum and vortexing. Tubes were allowed to stand at room temperature for $60 \mathrm{~min}$, after which they were cooled in an icewater bath for $30 \mathrm{~min}$. A charcoal suspension $(200 \mu \mathrm{l})$ was added to each tube and centrifuged, vortexed briefly, and then allowed to stand in the ice bath for $10 \mathrm{~min}$. The tubes were again centrifuged to sediment the charcoal. A 500- $\mu$ l sample of the supernatant from each tube was added to scintillation cocktail for counting. Values for DHT and total steroids, or T + DHT, were calculated from the appropriate standard curve. The antisera used has a $45 \%-50 \%$ cross-reactivity with DHT. Hence, $\mathrm{T}$ was calculated by the following equation:

$$
\mathrm{T}+\text { total }(\mathrm{T}+\mathrm{DHT})-y(\mathrm{DHT}) / 100,
$$

where $y=$ the percentage of cross-reaction of DHT that was obtained 
from the ratio of the midranges (pg steroid corresponding to $\mathrm{Co} / \mathrm{Cx}=$ 2) of the T and DHT standard curves. Standard-curve $R^{2}$ values were .78 and .84 for $T$ and DHT, respectively.

\section{RESULTS}

The mean $\mathrm{T}$ level of the group was $6.06 \mathrm{ng} / \mathrm{ml}(S D=$ $1.51)$; the mean DHT level was $1.77 \mathrm{ng} / \mathrm{ml}(S D=0.60)$. These are normal values for healthy young men. Correlation coefficients of T, DHT, and DHT/T measures to SIBT and MPFB scores and to the mean $z$ scores for the SIBT + MPFB ("spatial $z$ ") were computed. None of the correlations of DHT or T to spatial measures was significant. The DHT/T measure, however, was correlated with the MPFB score $(\varrho=-.27, p<.041)$. This indicates that the lower DHT was in relation to $\mathrm{T}$, the better the performance on the MPFB.

Examination of the scatterplots of these variables proved most interesting with respect to DHT/T. Figure 1 shows the scatterplots for DHT/T with MPFB (top left panel) and with the spatial $z$ score (top right panel). It is apparent that the great majority of DHT/T values were near the normal value of the sample (the normal value of DHT being approximately one-third that of $T$ ). It can also be seen that the spatial test scores of these "normal-value subjects" are distributed rather evenly from low to high. The 12 subjects whose values were more deviant from the normal DHT/T value, however, all scored from relatively high to quite high on the spatial tasks. Thus, there is substantial "predictability" of the spatial scores of subjects who are deviant in DHT/T values (i.e., they do not score low). There is no predictability for those whose $\mathrm{DHT} / \mathrm{T}$ values were quite near the normal value.
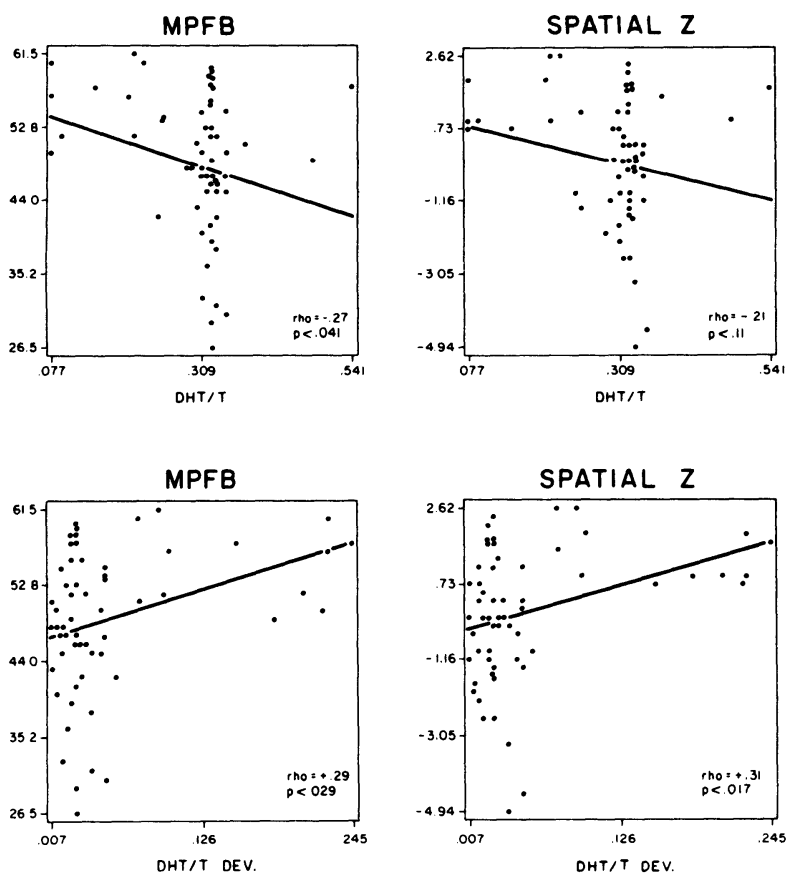

Figure 1. MPFB scores plotted against DHT/T values (upper left panel) and against DHT/T deviation values (lower left panel), and spatial $z$ scores plotted against DHT/T values (upper right panel) and against DHT/T deviation values (lower right panel).
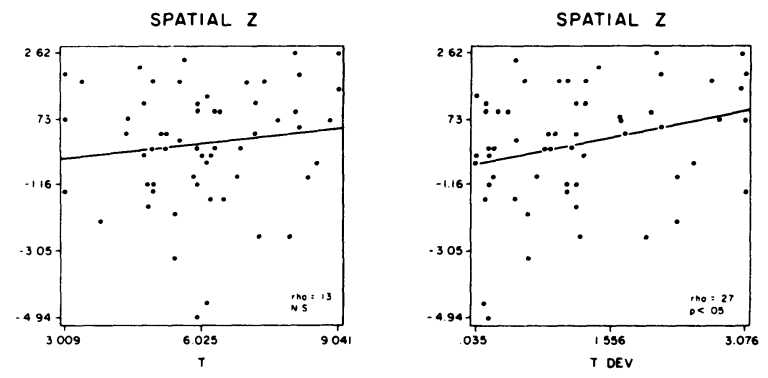

Figure 2. Spatial $z$ scores plotted against $T$ values (left panel) and against $T$ deviation values (right panel).

If one converts the DHT/T scores to DHT/T deviation scores, without regard to sign, and correlates these with the ability test scores, two significant correlations are found. These are for MPFB $(\varrho=+.29, p<.029)$ and for the spatial $z$ score $(\varrho=+.31, p<.017)$. Scatterplots of these deviation measures against MPFB and spatial $z$ scores are shown in the lower left and lower right panels, respectively, of Figure 1. It can be seen that the relationships cannot be optimally captured via regression analysis, since there is no relationship between the hormone measures and the ability measures for about 46 of the subjects. Contrasting the mean scores of the 12 most deviant DHT/T subjects with those of the remaining subjects, however, yields highly significant differences for the MPFB $[t(56)=3.28, p<.002]$, the spatial $z$ score $[t(56)=3.91, p<.001]$, and the SIBT $[t(56)=3.02$, $p<.004]$. The mean score differences of the groups correspond to 1.25 standard deviations for the spatial $z$ score, 1.0 standard deviation for the MPFB, and 0.9 standard deviations for the SIBT.

Finally, examination of the scatterplots of DHT with ability measures showed no relationships, but a similar examination for $T$ suggested a relationship between the more deviant $T$ values and the spatial $z$ score. Figure 2 shows the scatterplot for these variables, the left panel being simply $T$ versus the spatial $z$ score and the right panel being deviations (without respect to sign) of $T$ scores from the group mean versus the spatial $z$ score. It can be seen in the latter panel that the most deviant subjects tended to score higher than those with more representative deviations from the mean $T$ value. The left panel shows that the most deviant subjects were more often high$\mathrm{T}$ than low-T subjects. A Spearman correlation of the scores shown in the right panel was significant $(\varrho=.27$, $p<.045)$. Interestingly, the DHT/T deviations and T deviations showed no significant intercorrelation $(e=$ $.01, p<.913$ ). These two measures, when combined as predictors of the spatial $z$ score, showed an adjusted $\mathbf{R}^{2}$ of $.147(p<.005)$.

\section{DISCUSSION}

The data of Broverman et al. (1964), Klaiber, Broverman, and Kobayashi (1967), and Shute et al. (1983) argue for a negative relationship of androgens to spatial ability in males. Christensen and Knussman (1988), however, found low positive correlations between andro- 
gens and spatial ability. The present data failed to show any significant correlations of either T or DHT with spatial task performances. The directions of the nonsignificant correlations were, however, all positive.

Only Christensen and Knussman (1988) have looked at the relationship of this ratio to spatial ability, and they found it to be negatively related to spatial ability measures. In the present study, this measure showed a significant negative relationship to the MPFB. The negative relationships mean that the lower DHT was in relation to $\mathrm{T}$, the higher the ability test scores.

The most interesting feature of the present results, however, is that subjects whose DHT/T measures were most deviant from the overall DHT/T mean of the sample were consistently high scorers. The DHT/T deviation scores correlated significantly with both the MPFB and the spatial $z$ score. The 12 most deviant subjects scored markedly higher than the remainder of the subjects on the MPFB, SIBT, and the spatial $z$ score. A significant correlation was also observed between $T$ deviation scores and the spatial $z$ score, with the direction indicating that subjects with higher $\mathrm{T}$ deviations performed better on the combined spatial task measure. The DHT/T deviation and T deviation measures, together, accounted for $14.7 \%$ of the spatial $z$ score variance.

The finding of better spatial task performances by subjects whose hormone levels were "deviant" was, of course, not hypothesized and needs to be replicated. It is worth noting, however, that we recently completed a small-scale $(N=22)$ study of FSH and the same spatial ability measures described here. No relationship of FSH to the spatial ability measures was found. Again, however, deviation scores showed a significant relationship to both MPFB and spatial $z$ scores $(\varrho=+.54, p<.009$, and $e=+.49, p<.020$, respectively). In this instance, most of the "deviant" FSH subjects were high-FSH subjects.

Suggested explanations for the relevance of steroid levels to abilities have focused principally on the fact that steroids appear to elevate central norepinephrine (NE) levels through their ability to suppress monoamine oxidase levels (Broverman, Klaiber, Kobayashi, \& Vogel, 1968; Komnenich, Lane, Dickey, \& Stone, 1978). Broverman et al. (1968) suggested that higher NE levels are associated with higher levels of cortical activation, and that such activation is unfavorable for "perceptual restructuring" activities. However, most studies that have assayed steroids have not found significant negative relationships between steroid levels and spatial ability to begin with (Christensen \& Knussman, 1988; Gordon \& Lee, 1986; McKeever et al., 1987). Even within this model, it is difficult to envision how both deviantly low and deviantly high values of DHT/T could effect comparable enhancements of spatial performance.

The one previous finding that our results support is the negative relationship of DHT/T to some spatial ability measures (Christensen \& Knussman, 1988). Christensen and Knussman apparently did not look for possible nonlinear relationships. Our results, however, suggest that although DHT/T values correlate with MPFB scores, the deviation scores of DHT/T are more strongly related to spatial ability.

\section{REFERENCES}

Berenbaum, S. A., \& Resnick, A. (1982). Somatic androgyny and cognitive abilities. Developmental Psychology, 18, 418-423.

Broverman, D. M., Broverman, I. K., Kobayashi, Y., \& Palmer,
R. (1964). The automatization cognitive style and physical development. Child Development, 35, 1343-1359.

Broverman, D. M., Klaiber, E. L., Kobayashi, Y., \& Vogel, W. (1968). Roles of activation and inhibition in sex differences in cognitive abilities. Psychological Review, 75, 23-50.

Broverman, D. M., Vogel, W., Klaiber, E. L., Majcher, D., SheA, D., \& PAUL, V. (1981). Changes in cognitive task performance across the menstrual cycle. Journal of Comparative \& Physiological Psychology, 95, 646-654.

Christiansen, K., \& Kussman, R. (1988). Sex hormones and cognitive functioning in men. Neuropsychobiology, 18, 27-36.

Gordon, H. W., LEE, P. A. (1986). A relationship between gonadotropins and visual-spatial function. Neuropsychologia, 24, 563-576.

Hakstian, A. R., \& Cattell, R. B. (1976). Comprehensive ability battery. Champaign, IL: Institute for Personality and Ability Testing.

Hampson, E., \& KimuRA, D. (1988). Reciprocal effects of hormonal fluctuations on human motor and perceptual-spatial skills. Behavioral Neuroscience, 3, 456-459.

HARRIS, L. J. (1981). Sex-related variations in spatial skill. In L. Liben, N. Newcombe, \& A. Patterson (Eds.), Spatial representation and behavior across the life span (pp. 83-125). New York: Academic Press.

Klaiber, E. L., Broverman, D. M., Kobayashi, Y. (1967). The automatization cognitive style, androgens, and monoamine oxidase. Psychopharmocologia, 11, 320-336.

Komnenich, P., LANe, D. M., Dickey, R. P., \& Stone, S. C. (1978). Gonadal hormones and cognitive performance. Physiological Psychology, 6, 115-120.

Likert, R., \& QuASha, W. H. (1941). The Revised Minnesota Form Board. New York: The Psychological Corporation.

MACCOBY, E. E., \& JACKLIN, C. N. (1974). The psychology of sex differences. Stanford, CA: Stanford University Press.

McGee, M. G. (1979). Human spatial abilities: Psychometric studies and environmental, genetic, hormonal, and neurological influences. Psychological Bulletin, 86, 889-918.

McKeever, W. F., Rich, D. A., Deyo, R. A., \& Conner, R. L. (1987). Androgens and spatial ability: Failure to find a relationship between testosterone and ability measures. Bulletin of the Psychonomic Society, 25, 438-440.

Petersen, A. C. (1976). Physical androgyny and cognitive functioning in adolescence. Developmental Psychology, 12, 524-533.

SHIPLEY, W. C. (1940). A self-administering scale for measuring intellectual impairment and deterioration. Journal of Psychology, 9, 371-377.

Shute, V. J., Pellegrino, J. W., Hubert, L., \& Reynolds, R. W. (1983). The relationship between androgen levels and human spatial abilities. Bulletin of the Psychonomic Society, 21, 465-468.

STAFFord, R. (1961). Sex differences in spatial visualization as evidence of sex-linked inheritance. Perceptual and Motor Skills, 13, 391-393.

Thurstone, T. G. (1965). Primary mental abilities. Chicago: Science Research Associates.

(Manuscript received October 24, 1989.) 